Revista Mexicana de Economía y Finanzas, Vol. 9, No. 2, (2014), pp. 105-124

\title{
FACTORES QUE INCIDEN EN UNA MAYOR TRANSPARENCIA DE GOBERNANZA CORPORATIVA EN EMPRESAS COTIZADAS LATINOAMERICANAS
}

\section{Guadalupe del Carmen Briano Turrent*}

Facultad de Contaduría y Administración, Universidad Autónoma de San Luis Potosí

(Recibido 16 abril de 2014, aceptado 22 de junio de 2014)

\section{Resumen}

Bajo el enfoque de la teoría institucional, nuestro trabajo realiza un estudio comparativo del nivel de transparencia en gobierno corporativo de empresas cotizadas Latinoamericanas en el periodo de 2004-2010. Por otro lado, identifica aquellos factores institucionales formales que inciden en la transparencia de gobernanza empresarial. Los resultados ponen de manifiesto que el sistema legal, el tamaño y composición del consejo, la presencia del comité de auditoría, el tamaño de la empresa, el nivel de endeudamiento y el sector industrial inciden significativamente en el nivel de transparencia en gobierno corporativo en la región Latinoamericana.

\section{Abstract}

Under the institutional theory approach, our work aims to obtain a comparative study of corporate governance transparency level on Latin American listed companies during 20042010 period. On the other hand, to identify those formal institutional factors that have a significant influence on corporate governance disclosure. The results indicate that legal system, board size, board composition, audit committee, size of the company, leverage, and industry type influence on corporate governance transparency on Latin American region.

Clasificación JEL: E43, G3, D20, E65, F31.

Palabras clave: Transparencia, Gobierno corporativo, Teoría institucional, Latinoamérica, Países emergentes.

* Sierra Leona No. 550. Lomas 2a. Sección. San Luis Potosí, S.L.P., México. Correo electrónico: guadalupe.briano@uaslp.mx 


\section{Introducción}

La globalización, la internacionalización de los mercados y la desregulación en los países hacen que el tema de gobierno corporativo [GC] sea un tema de gran relevancia en la última década, despertando un creciente interés en la literatura comparativa internacional. Por otro lado, los recientes escándalos financieros que tuvieron lugar en EE.UU., Europa y Latinoamérica, han avivado el interés por el tema. Así, Mercer (2004) y Hodge et al. (2006) afirman que la credibilidad de la información es un elemento esencial en el buen gobierno de una sociedad. La transparencia corporativa ha sido adoptada como uno de los principios rectores del buen gobierno por organismos internacionales como la Organización para el Desarrollo y Cooperación Económicos [OCDE].

En Latinoamérica, la protección legal del inversionista es débil comparada con Europa y EE.UU., por lo que la concentración de la propiedad es el modo predominante de gobierno (Djankov, La Porta y López-de-Silanes, 2008). Sin embargo, en materia de transparencia y GC se ha visto un importante avance en los últimos años, especialmente en las cuatro economías más importantes de Latinoamérica, quienes concentran la mayor parte de la capitalización bursátil: Argentina, Brasil, Chile y México (Diamandis y Drakos, 2011). Latinoamérica se ha adherido de forma voluntaria a las prácticas y políticas de GC para cubrir parcialmente la deficiencia legal qu/e opera en el entorno (Garay y González, 2008). Así, la OCDE emite en 1999, los "Principios de la OCDE para el gobierno de las sociedades", convirtiéndose desde entonces en referencia internacional para el GC y la base para diversas iniciativas de reforma, tanto gubernamentales como del sector privado (OCDE, 2004).

El creciente interés público en la transparencia corporativa ha sido reflejado en la nueva regulación emitida por diferentes organismos internacionales. Así, encontramos los principios de buen gobierno emitidos por la OCDE en 2004, entre los cuales destaca el de divulgación de datos y transparencia. Otra ley que ha sido un referente en diferentes países y que recoge la normativa referida a la importancia de la tecnología, y en especial de Internet, para el GC, es la Ley de Sabarney Oxley Act, ${ }^{1}$ en cuya sección 409 establece la obligación de que las compañías informen en tiempo real de los hechos que afecten significativamente su posición financiera y económica. En el caso de Latinoamérica se han tomado como referencia los principios de buen gobierno y los requerimientos mínimos de divulgación de información emitidos por la OCDE, así como los contenidos en los códigos de mejores prácticas corporativas.

El presente estudio realiza un comparativo del nivel de transparencia en GC en países emergentes Latinoamericanos e identifica los factores institucionales formales (referidos al sistema legal y las dimensiones de GC) que motivan a las empresas cotizadas a ser más transparentes en el mercado. Latinoamérica ofrece un escenario ideal para el estudio, ya que se caracteriza por una débil protección a los accionistas minoritarios y una alta concentración de la propiedad, la cual actúa como sustituto en la ausencia de un sistema legal fuerte (Matin de Holan y Sanz, 2006).

El resto del trabajo está estructurado de la siguiente forma: En la primera sección se desarrolla una revisión de la literatura. La segunda describe la

\footnotetext{
1 Puede consultarse el texto legal en:http://www..law.uc.edu/CCL/SOact/toc.html
} 
metodología y diseño de la investigación. La tercera sección discute los resultados del análisis estadístico. Finalmente se presentan las conclusiones, limitaciones del estudio y futuras líneas de investigación.

\section{Revision de la Literatura}

En este estudio se adoptó la teoría institucional para analizar los factores formales (sistema legal y dimensiones de gobierno corporativo) que influyen en el nivel de transparencia de gobierno corporativo. La literatura previa ha mostrado que otras teorías como la teoría de la agencia, teoría de los stakeholders y la teoría de la legitimidad han explicado el nivel de transparencia corporativa. Sin embargo, el marco conceptual de la teoría institucional es mucho más amplio y profundo (Sobhani, Zainuddin, Amran, y Baten, 2011). Por otra parte, este enfoque teórico es el más adecuado para explicar la divulgación de información en gobierno corporativo de las empresas cotizadas en países emergentes Latinoamericanos, ya que estos se caracterizan por un fuerte vínculo entre las estructuras de gobierno corporativo y el desarrollo institucional. Globerman y Shapiro (2003) observaron que las instituciones formales - la normativa y leyes, la transparencia y la rendición de cuentas - fortalecen la estructura de gobierno y atrae más inversión extranjera.

En este sentido, la hipótesis general del presente estudio se ha basado en la teoría institucional, la cual se define como un conjunto de reglas formales e informales que inciden en la actividad empresarial (North, 2005). El entorno institucional se integra de tres pilares: el regulatorio, el normativo y el culturalcognitivo. El pilar de la regulación se basa en la configuración de las reglas, el seguimiento, la recompensa y el castigo. El pilar normativo se centra en los valores, las normas y la tradición que introducen una dimensión en la vida social. Por último, la dimensión cultural-cognitiva es el conjunto de creencias compartidas que constituyen la naturaleza de la realidad social y los marcos a través del cual el sentido se hace (Scott, 2008). En este sentido, tanto las instituciones formales como las iniciativas del gobierno, las leyes y las instituciones informales como la cultura y la estrategia conforman los antecedentes de la acción mediante la definición de las "reglas del juego" (Boliari y Topyan, 2007). De acuerdo con DiMaggio y Powell (1983), la teoría institucional señala que las empresas tienden a incorporar las normas y reglas externas en sus operaciones y estructuras con el fin de ganar legitimidad y aceptación social a través de la transparencia corporativa. De este modo, todas las formas de las instituciones que gestionan las interacciones humanas a través de los procesos cognitivos, normativos y regulatorios influyen en la toma de decisiones empresarial (Treviño, Thomas y Cullen 2008).

Con respecto a las variables que tienen una incidencia significativa en el nivel de transparencia, Jaggi y Low (2000), afirman que las dimensiones políticas, sociales y económicas de un país inciden en ésta. Así, Bushman et al. (2004) exploran la relación existente entre la transparencia corporativa y tres aspectos del regimen legal/judicial: origen legal, eficiencia del sistema judicial y el grado de protección de las patentes. Por su parte, Durnev y Han Kim (2005) revelan que las prácticas de GC y divulgación de información muestran una amplia variación entre los países, la cual disminuye con la fortaleza en la protección legal del inversor. Con respecto a las dimensiones de GC, Ho y Wong (2001) identifican que la proporción de consejeros independientes, la presencia 
del comité de auditoría, la dualidad COB-CEO y el porcentaje de miembros familiares en el consejo inciden en el nivel de transparencia corporativa. En esta misma línea, Tsamenyi et al., (2007) y Mangena y Tauringana (2007), sostienen que la concentración de la propiedad y la presencia de un comité de auditoría tienen una influencia significativa en la transparencia corporativa. En el contexto Latinoamericano, Silva et al. (2008) sostienen que el nivel de endeudamiento y el tamaño de la empresa influyen significativamente en la transparencia corporativa. Para el caso específico de México, el tema de GC solamente se ha relacionado con el desempeño corporativo de la empresa Chong et al. (2009), mientras que Leal y Carvalhal (2005) y Lefort y Walker (2005) han analizado los casos de Brasil y Chile.

$\mathrm{Al}$ tenor de lo expuesto, la hipótesis general de la presente investigación sostiene que los factores institucionales formales referidos al sistema legal y dimensiones de gobierno corporativo, inciden positivamente en el nivel de transparencia de gobierno corporativo en las empresas de mayor liquidez bursátil de países emergentes Latinoamericanos.

\subsection{Desarrollo del modelo y medición de las variables}

Para comprobar la hipótesis propuesta, se ha estimado el siguiente modelo de regresión lineal múltiple. La variable dependiente es el índice de transparencia en gobierno corporativo $(e-I T G C)$, mientras que las variables explicativas son el sistema legal y las variables de gobierno corporativo (tamaño del consejo, independencia del consejo, concentración de la propiedad, dualidad COB-CEO, participación femenina en el consejo y presencia del comité de auditoría). Se han incluido como variables de control el tamaño de la empresa, la edad de la empresa, el sector industrial, la rentabilidad y el nivel de endeudamiento (Vander Bauwhede y Willekens, 2008). El cuadro 1 resume la definición y signo esperados para cada una de las variables utilizadas en el modelo.

$$
\begin{aligned}
e-I T C & =\beta_{0}+\beta_{1} W_{\text {GI }}+\beta_{2} \text { Board_Size }_{j}+\beta_{3} \text { Board_Ind }_{j}+\beta_{4} \text { Own }_{j} \\
& +\beta_{5} \text { Dual }_{j}+\beta_{6} \text { Audit }_{j}+\beta_{7} \text { Gender }_{j}+\beta_{8} \text { Lev }_{j}+\beta_{9} \text { Ln_Size }_{j} \\
& +\beta_{10} \operatorname{Ln}_{A} \text { ge }_{j}+\beta_{11} \text { ROA }_{j}+\beta_{12} \text { Ind }_{j}+\varepsilon_{j}
\end{aligned}
$$

\subsubsection{El sistema legal}

La relevancia que tiene el sistema legal en el nivel de protección de los inversionistas y en la estructura de GC de las empresas fue puesta de manifiesto por La Porta et al. (1998). El sistema legal tiene una influencia significativa en el desarrollo de los sistemas contables y en el crecimiento y desarrollo económicos (Jaggi y Low, 2000). El sistema legal en países de tradición anglosajona cuenta con una tasa de cumplimiento legal mayor y, por tanto, se incrementa la protección de los derechos de accionistas y acreedores; mientras que en países que disponen de una menor protección legal a los inversionistas, presentan una elevada concentración de la propiedad y se recurre a otros mecanismos de buen gobierno para generar la confianza en el mercado (La Porta et al., 2006).

De acuerdo con Leuz et al. (2003) la aplicación de la ley se ha medido a través de tres variables: a) la eficiencia del sistema judicial, b) evaluación del estado de derecho, y c) el índice de corrupción. Bajo este enfoque, hemos de 
adoptar el Índice Mundial de Gobierno (WGI), publicado anualmente por el Banco Mundial y el cual recoge las tradiciones e instituciones a través de las cuales la autoridad de un país es aplicada, incluyendo el proceso a través del cual el gobierno es elegido y controlado, su capacidad para formular e implementar efectivamente las políticas y la interacción entre las instituciones económicas y sociales. Este índice se integra de seis dimensiones de gobierno (voz y rendición de cuentas, estabilidad política y ausencia de violencia, efectividad de gobierno, calidad regulatoria, estado de derecho, y control de la corrupción). De acuerdo con Kaufmann et al. (2010), el WGI permite realizar comparaciones entre países y a través del tiempo. En este sentido esperamos que en economías emergentes Latinoamericanas la fortaleza de la ley influya en un mayor nivel de transparencia en gobierno corporativo. Dado lo anterior el presente estudio sostiene una relación positiva entre el WGI del país y el índice de transparencia en GC de empresas cotizadas latinoamericanas.

Cuadro 1. Resumen de variables y anotaciones en el modelo de regresión

\begin{tabular}{|c|c|c|c|c|}
\hline Variable & $\begin{array}{l}\text { Descripción de la } \\
\text { yariable }\end{array}$ & $\begin{array}{l}\text { Notación en } \\
\text { el modele }\end{array}$ & Fuente & $\begin{array}{l}\text { Signa } \\
\text { espexad } \\
0\end{array}$ \\
\hline eITGC & $\begin{array}{l}\text { Índice de tranabsrencia } \\
\text { en Gobiemo Corporativ }\end{array}$ & e-ITGC & $\begin{array}{l}\text { Garay y Gonzalez, }(2008) \text {; } \\
\text { Leal y Carvalhal -ds-Silva, } \\
(2005) \text {; Gandia y Andrés, } \\
(2005)\end{array}$ & \\
\hline Sistema legal & $\begin{array}{l}\text { Indicador de gobieme } \\
\text { mundial }\end{array}$ & $\mathrm{WGI}_{4}$ & World Bank (2010) & + \\
\hline $\begin{array}{l}\text { Tamaño del } \\
\text { consejo }\end{array}$ & $\begin{array}{l}\text { Logaritmo natural del } \\
\text { númers de miembroa } \\
\text { en el conajo. }\end{array}$ & Boserd_size, & $\begin{array}{l}\text { Kent y Steward, (2008) } \\
\text { Baralko et al. (2006) } \\
\text { Willekens et al. (2005) }\end{array}$ & + \\
\hline $\begin{array}{l}\text { Independencia dal } \\
\text { conseja }\end{array}$ & $\begin{array}{l}\text { Proporción de } \\
\text { conajerps } \\
\text { independientea }\end{array}$ & Bosrd_jnd & $\begin{array}{l}\text { Chen y Jaggi (2000); Leung } \\
\text { x Howsitz (2004); Ajinkty } \\
\text { et al., 2005; Kent y Steward } \\
\text { (2008). }\end{array}$ & - \\
\hline $\begin{array}{l}\text { Estructurs de la } \\
\text { Propiedad }\end{array}$ & $\begin{array}{l}\text { Porcentaie de las } \\
\text { acciones compuna } \\
\text { sostenidas por los trea } \\
\text { principslea accionistas }\end{array}$ & Own & $\begin{array}{l}\text { Hope et al (2008); Barako } \\
\text { et al. (2006): Lefort y } \\
\text { Walker }(2005)\end{array}$ & - \\
\hline COB-CEO & $\begin{array}{l}\text { Variable dicotómical } \\
0,0\end{array}$ & Dual, & $\begin{array}{l}\text { Gandia (2008); Barakp et } \\
\text { al, }(2006 \text { : }\end{array}$ & - \\
\hline $\begin{array}{l}\text { Comité } \\
\text { de auditeris }\end{array}$ & $\begin{array}{l}\text { Presencia del comité } \\
\text { de auditoria (Variable } \\
\text { dicotómica l o } 0 \text { ) }\end{array}$ & Audil & $\begin{array}{l}\text { TWillelvens et al (2005). } \\
\text { Barakso et al. (2006) } \\
\text { Hoy Wong (2001) }\end{array}$ & + \\
\hline $\begin{array}{l}\text { Participación } \\
\text { femenins en al } \\
\text { conseia }\end{array}$ & $\begin{array}{l}\text { \% de mujerea en el } \\
\text { conasis de } \\
\text { adrninistración }\end{array}$ & Genden & $\begin{array}{l}\text { Jaggi y Low (2000); Hope } \\
(2003)\end{array}$ & - \\
\hline Endeudamieuth & $\begin{array}{l}\text { Endeudamiento = } \\
\text { denda a largo } \\
\text { plszoutotal de activos }\end{array}$ & Lev & $\begin{array}{l}\text { Jaggi y Low (2000) } \\
\text { Willekenset al (2005). }\end{array}$ & + \\
\hline $\begin{array}{l}\text { Tamaño de la } \\
\text { Empresa }\end{array}$ & $\begin{array}{l}\text { Logeritmo natural del } \\
\text { total de emplesdos }\end{array}$ & LnSizen 1 & Craven y Maraton (1999) & + \\
\hline
\end{tabular}


Cuadro 1. Resumen de variables y anotaciones en el modelo de regresión (continuación)

\begin{tabular}{|c|c|c|c|c|}
\hline Variable & $\begin{array}{l}\text { Descripción de la } \\
\text { sariable }\end{array}$ & $\begin{array}{l}\text { Notación en } \\
\text { el modela }\end{array}$ & Fuente & $\begin{array}{l}\text { Signo } \\
\text { esperad } \\
0\end{array}$ \\
\hline Reutabilidad & $\begin{array}{l}\text { Retorno de los activos, } \\
\text { (EBIT)/ yalor en libros } \\
\text { del total de activos }\end{array}$ & $\mathrm{ROA}_{11}$ & $\begin{array}{l}\text { Dye (1985 y } 1986 \text { ) } \\
\text { Verreschia (1983 y 1990x } \\
\text { Daropughy S Stoughton } \\
\text { (1990); Garay y González, } \\
\text { (2008). }\end{array}$ & + \\
\hline $\begin{array}{l}\text { Sector industrial } \\
\text { Añog }\end{array}$ & $\begin{array}{l}\text { Variable dummy (10 } \\
\text { sectorea induatriales) } \\
\text { Variable dummy } \\
\text { (periods 2004-2010) }\end{array}$ & $\begin{array}{l}\text { Ind, } \\
\text { Year, }\end{array}$ & GICS & \\
\hline
\end{tabular}

Fuente: Elaboración propia, basada en trabajos previos

\subsubsection{Independencia del consejo}

Fama y Jensen (1983), han reconocido que el consejo de administración es el mecanismo más importante de control en la estructura de gobierno de una empresa. En esta línea, Patelli y Prencipe (2007) afirman que la composición del consejo es uno de los principales mecanismos que mitiga el conflicto de agencia, y sostienen que +los miembros independientes son necesarios en el consejo para controlar las acciones de la gerencia. Los consejeros internos generalmente pertenecen al grupo familiar o directivo de la empresa, mientras que los consejeros externos carecen de un vínculo financiero o personal con la gestión de la empresa, además de sus honorarios y acciones (Rouf, 2011). Los consejeros independientes son más efectivos en la maximización del valor para los accionistas, y por tanto, pueden contribuir con un mayor conocimiento y experiencia. Bajo este enfoque, se ha asociado una relación significativa entre el nivel de divulgación de información y la proporción de consejeros independientes en diferentes contextos (Chen y Jaggi, 2000; Leung y Horwitz, 2004; Ajinkya et al., 2005; Kent y Steward, 2008). Al tenor de lo expuesto, se espera que una mayor proporción de consejeros independientes incremente el nivel de divulgación de GC en empresas cotizadas Latinoamericanas.

\subsubsection{Concentración de la propiedad}

Un factor importante que da forma al sistema de gobierno corporativo es la estructura de la propiedad, la cual se define como el grado de concentración que determina la distribución de poder y control corporativo, o bien como la proporción de acciones con derecho a voto que son de propiedad directa o indirecta de los familiares de los altos directivos o del consejo de administración (Owusu-Ansah, 1998). El grado de concentración de la propiedad determina la distribución del poder sobre el control corporativo (Shan y Mclver, 2011). De acuerdo con McKinnon y Dalimunthe (1993), cuando la estructura de propiedad es difusa, existe la necesidad de fortalecer el control, y consecuentemente una mayor divulgación de información corporativa para mantener la equidad de acceso a los accionistas minoritarios. En este sentido, Haniffa y Cooke (2002) sostienen que la estructura de la propiedad determina el nivel de transparencia corporativa, particularmente en aquellas empresas con una gran dispersión de su propiedad, en donde el equipo gerencial promueve mayor transparencia como 
una señal de su actuación en beneficio de los intereses del principal; mientras que en estructuras de propiedad muy concentradas los niveles de divulgación de información son más bajos. Diversos estudios ponen de manifiesto una relación negativa entre la concentración de propiedad familiar y el nivel de divulgación de información (Ho y Wong, 2001; Barako et al., 2006; Vander Bauwhede y Willekens, 2008; Gandía, 2008). En este sentido, en empresas con una alta concentración de propiedad, la transparencia en gobierno corporativo será menor, dado que la información puede ser transferida directamente a través de los canales informales, o simplemente porque existe una mayor alineación de intereses, lo cual reduce la necesidad de un mejor gobierno sobre los accionistas minoritarios.

\subsubsection{Dualidad COB-CEO}

La eficacia y eficiencia del consejo de administración se puede ver comprometida si las posiciones del presidente del consejo y el director general son sostenidas por la misma persona (Blackburn, 1994). De acuerdo con Haniffa y Cooke (2002), la separación entre ambas posiciones ayuda a mejorar la calidad de la supervisión y a reducir las ventajas obtenidas por la retención de información, mejorando la calidad en la divulgación de información corporativa. Bajo esta premisa, la concentración de poder de ambas posiciones está asociada a erosionar la independencia del consejo de administración y a reducir la divulgación y calidad de la información corporativa (Simon y Wong, 2001). Gandía (2008) sostiene que la dualidad COB-CEO va en detrimento de la empresa, mientras que la separación de éstas conduce a un aumento en la eficacia del consejo de administración y promueve una mayor independencia entre el consejo de administración y la dirección general (Fama y Jensen, 1983; Baysinger y Hoskisson, 1990). Dado lo antes expuesto, se espera una relación negativa entre la dualidad COB-CEO y el nivel de transparencia corporativa en GC para las empresas que cotizan en Latinoamérica.

\subsubsection{Comité de auditoría}

El consejo de administración, generalmente delega responsabilidades trascendentes al comité de auditoría, el cual representa una parte esencial del gobierno corporativo, encargándose de la supervisión y control de los objetivos organizacionales, lo cual a su vez permite la divulgación de información veraz y oportuna dirigida a todos los grupos de interés de la empresa (Willekens et al., 2005). Históricamente los comités de auditoría surgieron como un mecanismo de supervisión voluntario con el objetivo de mejorar el flujo de información entre el principal y el agente (Collier, 1997; Rouf, 2011). La investigación empírica previa pone en evidencia una asociación positiva entre la existencia del comité de auditoría y las prácticas de divulgación de información corporativa (Barako et al., 2006; Ho y Wong, 2001). Por ello se pretende comprobar que el nivel de transparencia en GC es mayor para aquellas empresas que cuentan con un comité de auditoría.

\subsubsection{Participación femenina en el consejo de administración}

En los últimos años, el tema de la diversidad de género en los negocios ha recibido atención en el mundo académico y la prensa. De acuerdo con Gul, Srinidhi y Ng (2011) la diversidad de género en el consejo, podría mejorar la calidad de los debates en el consejo e incrementar la capacidad de éste en la supervisión del proceso de divulgación de información corporativa diseminada 


\section{Nueva Época REMEF (The Mexican Journal of Economics and Finance)}

por la empresa en sus memorias anuales. Algunos estudios han analizado la presencia de la mujer en el consejo y su impacto en los resultados financieros de la empresa (Francoeur et al., 2008; Adams y Ferreira, 2004). Por su parte, son diversos los estudios que sugieren que la diversidad de género en el consejo está asociada a una mayor calidad de las deliberaciones del consejo y a una efectiva comunicación (Huse y Solberg, 2006; McInerney-Lacombe, Bilimoria y Salipante, 2008), lo cual incrementa la diseminación de información hacia los inversionistas. En este contexto es de esperar que la participación femenina en el consejo influya positivamente en la transparencia de gobierno corporativo en empresas cotizadas Latinoamericanas.

\subsubsection{Características de la empresa}

Son diversas las variables corporativas que pudieran afectar el nivel de transparencia corporativa en las empresas cotizadas. La literatura previa ha puesto en evidencia que las empresas con un mayor endeudamiento están generalmente bajo un escrutinio más estricto por parte de los acreedores, por lo que las empresas divulgarán mayor información sobre su gestión (Jaggi y Low, 2000; Oyelere et al., 2003). Por su parte, la edad de la empresa es una variable que representa la asimetría de información entre los inversionistas y se mide como el número de años en que la firma ha cotizado en la bolsa de valores del país de origen (Gandía, 2008). En este sentido, la asimetría de información se relaciona negativamente con la edad de la empresa, por lo cual las empresas nuevas tienen mayores incentivos para combatir el escepticismo y aumentar la confianza de los inversores (Haniffa y Cooke, 2002). Diversos estudios han encontrado que el tamaño de la empresa tiene una influencia significativa sobre la divulgación de información (Barako et al., 2006; Bonsón y Escobar, 2006). Las empresas grandes en comparación con las pequeñas, están más motivadas a divulgar mayor información por contar con un mayor número de accionistas y stakeholders que requieren de información más amplia y detallada (Jaggi y Low, 2000). Esta variable es utilizada como una aproximación de los costos de propiedad, producción y políticos, y se mide frecuentemente como el logaritmo natural del total de los activos (Watts y Zimmerman, 1986; Lang y Lundholm, 1993). Por otro lado, la rentabilidad incide en el nivel de transparencia corporativa. De acuerdo con Inchausti (1997), las empresas más rentables harán un mayor uso de la información para obtener ventajas competitivas, mientras que aquellas empresas con bajo desempeño diseminan menos información. Y aunque no existe un consenso de la dirección de la relación entre ésta y la divulgación de información, algunos estudios revelan que las compañías más rentables son más transparentes hacia el mercado (Verrecchia, 1990; Darrough y Stoughton, 1990). Finalmente diversos estudios han asociado significativamente al sector industrial con la transparencia corporativa (Gandía, 2008; Bonsón y Escobar, 2006; Ettredge et al., 2001; Oyelere et al., 2003).

\section{Metodología y modelo empírico}

\section{1. Índice de transparencia en gobierno corporativo}

La herramienta más utilizada para medir la transparencia corporativa es la elaboración de índices de divulgación (Gandía y Andrés, 2005). La mayor parte de los estudios empíricos se han orientado hacia el estudio y análisis de contenido de los informes anuales de las empresas (Mir et al., 2009). El informe anual es considerado un medio relevante mediante el cual la com- 
pañía difunde información corporativa (Depoers, 2000). Otros estudios se han referido a la información divulgada en los sitios web de las empresas (Che Haat et al., 2008; Garay y González, 2008; Gandía, 2008; Bonsón y Escobar, 2006; Vander Bauwhede y Willekens, 2008). Para ello, se construyó un índice de transparencia en gobierno corporativo, en cuya composición se tomaron en cuenta trabajos previos realizados y la normativa y códigos de buen gobierno de cada país, así como los principios emitidos por la OCDE (2004). El índice se integró de 43 elementos, los cuales a su vez se categorizaron en cuatro subíndices: 1) composición y desempeño del consejo de administración, referidos a la misión y principales funciones del consejo, su independencia y estructura, los procedimientos de selección, participación accionarial y remuneración de los consejeros, así como los comités de apoyo al consejo; 2) derechos de los accionistas, relacionado al proceso de voto de los accionistas, las estructuras piramidales, asamblea y acuerdos entre accionistas; 3 ) ética y conflictos de interés, enfocado a los conflictos de interés y transacciones entre partes relacionadas, sanciones y multas, composición del accionariado; y 4) otra información relacionada con el buen gobierno, el cual se centró en la adopción de principios contables internacionales, la contratación del auditor externo, prácticas de buen gobierno y desempeño financiero.

Para calcular el valor del índice se adoptó la metodología propuesta por Gandía y Andrés (2005), tomando en cuenta la información mostrada en el cuadro 1. En concreto si la empresa reveló cada uno de los ítems, se le asignó el valor de 1 y 0 en caso contrario. Se consideraron dos índices de transparencia en gobierno corporativo: 1) el relacionado con la información divulgada en los sitios web de las empresas, y 2) el que cuantificó la información difundida en los informes anuales de los últimos siete años. Dado que los contenidos analizados se agruparon en cuatro dimensiones, en primer lugar se calcularon los índices parciales en cada uno de ellos, para posteriormente agregarlos para obtener el índice global. Los índices parciales son el cociente entre la suma de los puntos obtenidos respecto a los puntos totales que podía haber obtenido la empresa si hubiese difundido el máximo de ítems. Cada subíndice se obtuvo de la siguiente forma:

$$
e-I T G C^{p}=\frac{\text { Número de elementos divulgados en el apartado }}{\text { Total de elementos que se podrían obtener }}
$$

$\mathrm{El} e-I T G C$ global se define operativamente de la siguiente forma:

$$
e-I T G C^{T}=I_{i}^{p} \mathrm{xP}_{i}^{t}
$$

Donde

$e-I T G C^{T}=$ Valor global del índice.

$e-I T G C^{p}=$ Valor del subíndice en base a las dimensiones que integran el $e-I T G C^{T}$.

$I^{p}=$ Proporción que representa el subíndice " $i$ " respecto al número total de puntos del índice global.

El índice total, no se obtuvo como una media simple de los diferentes subíndices, sino como una media ponderada. De acuerdo a esta metodología, tanto los subíndices como el índice global podrán obtener un valor comprendido entre 0 y 1 puntos. 


\section{Nueva Época REMEF (The Mexican Journal of Economics and Finance)}

\subsection{Muestra de estudio}

La investigación se centró en las empresas de mayor liquidez bursátil en Argentina, Brasil, Chile y México. Los índices bursátiles reflejan el rendimiento del mercado en función de las variaciones de precio de la canasta de instrumentos que los conforman. En Argentina, el índice Merval es el más difundido del mercado accionario local; se compone de 17 acciones seleccionadas de acuerdo a criterios que ponderan su liquidez y su capitalización en el mercado de valores (Reuters, 2011). En Brasil, el indicador más importante del desempeño medio de las cotizaciones del mercado de acciones brasileño es el Ibovespa. Su relevancia proviene del hecho que éste retrata el comportamiento de los principales papeles negociados en la Bolsa de Valores de Brasil, las cuales representan el $80 \%$ del volumen negociado durante los últimos doce meses (BM\&FBOVESPA, 2011). En Chile, el índice más conocido es el IPSA (Índice Precio Selectivo de Acciones), el cual se integra de las 40 sociedades con mayor participación bursátil (Bolsa de Comercio, 2011). Para el caso de México, se han seleccionado las empresas que pertenecen al Índice de Precios y Cotizaciones (IPyC), el cual representa el mercado accionariado en su conjunto, en función de las variaciones de precios de una selección de acciones (o muestra) balanceada, ponderada y representativa del conjunto de acciones cotizadas en la Bolsa Mexicana de Valores.

La muestra de empresas se extrajo de los sitios web de cada una de las Bolsas de Valores en cada país bajo estudio. Para efectos de análisis, se excluyeron las empresas que pertenecen al sector bancario y financiero, dado que su regulación es más estricta y son sujetas de un mayor escrutinio en términos de divulgación de información (Garay y González, 2008). Para fines de agrupación, las empresas se analizaron por país y sector de actividad económica de acuerdo a la clasificación Global Industry Classifications Standards [GICS] (Bhojraj et al., 2003). La información financiera se obtuvo de los informes anuales y sitios web de cada empresa. La muestra final de estudio se compuso de 129 empresas. El periodo de análisis fue de 2004 a 2010, lo cual nos arrojó un total de 832 observaciones. Véase cuadro 2.

Cuadro 2. Número de empresas que integran la muestra de estudio

\begin{tabular}{|l|c|c|c|}
\hline Pais & $\begin{array}{c}\text { Número de } \\
\text { empresas } \\
\text { (incluidas } \\
\text { las empresas } \\
\text { financieras) }\end{array}$ & $\begin{array}{c}\text { Número de } \\
\text { empresas (no } \\
\text { inchidas las } \\
\text { empresas } \\
\text { financieras) }\end{array}$ & $\begin{array}{c}\text { Muestra de } \\
\text { estudio final }\end{array}$ \\
\hline Argentina & 17 & 12 & 10 \\
\hline Brasil & 63 & 58 & 55 \\
\hline Chile & 40 & 34 & 32 \\
\hline México & 35 & 31 & 32 \\
\hline $\begin{array}{l}\text { Total } \\
\text { empresas }\end{array}$ & 155 & 135 & 129 \\
\hline Total obs & & & 832 \\
\hline
\end{tabular}

Fuente: Elaboración propia. 
Con respecto a la distribución sectorial encontramos que para el caso de México la composición se dividió en dos grupos principales, bienes de consumo (30.3\%) y productos básicos de consumo (34.6\%), en Argentina son los sectores de materiales $(32.3 \%)$ y productos básicos de consumo $(21.5 \%)$ y empresas de servicios públicos $(27.7 \%)$ los más importantes. Por su parte, en Chile existe una distribución homogénea entre los sectores industriales de materiales, industrial, bienes de consumo, productos básicos de consumo y empresas de servicios públicos. Finalmente, Brasil mostró un mayor porcentaje en los sectores de materiales y empresas de servicios públicos.

\section{Análisis de resultados}

\subsection{Estadísticos descriptivos}

El término medio del e-ITGC fue de $72 \%$ en México, $51 \%$ en Argentina, $59 \%$ en Chile, y $61 \%$ en Brasil. Se observó que las empresas mexicanas concentraron sus esfuerzos de difusión discrecional en el subíndice de ética y conflictos de interés (88\%), seguido de la composición y desempeño del consejo $(76 \%)$. Argentina se centró al igual que México en la ética y conflictos de interés y en otra información relacionada con gobierno corporativo. Chile por su parte, obtuvo mayor puntuación para la ética y conflictos de interés $(74 \%)$ y otra información relacionada con el buen gobierno (73\%). Finalmente, Brasil se concentró en otra información relacionada con el buen gobierno (75\%) y en la ética y conflictos de interés. Asimismo los países han incrementado su nivel de divulgación de información a lo largo del periodo analizado. En relación a los sectores industriales, se encontró que el sector que divulgó más información es el de energía (71\%), seguido por el industrial (65\%), cuidado para la salud $(65 \%)$ y el de consumo (63\%). Mientras que los que menos divulgaron fueron los sectores de tecnologías de información (49\%) y empresas de servicios públicos (51\%).

El cuadro 3 resume los estadísticos descriptivos de las variables analizadas durante el periodo 2004-2010. El indicador mundial de gobierno (WGI) obtuvo una media $48 \%$ para México, $41 \%$ para Argentina, $84 \%$ para Chile y $52 \%$ en Brasil. El promedio del tamaño del consejo fue de 13 para México, 10 para Argentina, 8 para Chile y 9 para Brasil. Por su parte, la proporción de consejeros independientes fue de $48 \%$ en México, 27\% en Argentina, 35\% en Chile y $30 \%$ en Brasil. Con respecto a la dualidad COB-CEO, se observó que México y Argentina obtuvieron un porcentaje menor $\left(55.8 \%^{6}\right.$ y $55.4 \%$, respectivamente) con respecto' a Chile (95.2\%) y Brasil (78.1\%). La participación femenina en los consejos de administración es mínima en los países Latinoamericanos, siendo Brasil el que obtiene un mayor porcentaje (6\%), seguido de México con un $4 \%$ y Chile con un $2 \%$.

En cuanto a la presencia del comité de auditoría, se observó que el 100\% de las empresas de México y Argentina han constituido este Comité, en tanto que en Chile solamente el $28.8 \%$ de las empresas lo han adoptado. Particularmente en Chile, existen otros órganos de control como el directorio o el consejo fiscal. En Brasil el 93.2\% de las empresas ha institucionalizado el comité de auditoría. Con respecto a las acciones en manos de los accionistas controladores (OWN), la media para México fue de $54 \%$, Argentina obtuvo un $53 \%$, Chile un $58 \%$ y Brasil un $55 \%$. El nivel de endeudamiento (Lev) registrado para las empresas mexicanas fue de $18 \%$, mientras que para las empresas cotizadas en Argentina 
116 Nueva Época REMEF (The Mexican Journal of Economics and Finance)

de $19 \%, 27 \%$ en Chile y $31 \%$ para Brasil. La edad de la empresa obtuvo un promedio de 46 años en México, 47 en Argentina, 62 en Chile y 41 en Brasil. Con respecto al tamaño de la empresa (Ln de activos totales), México obtuvo una media de 8.18 para México, 7.57 en Argentina, 7.76 en Chile y 8.68 en Brasil. Por su parte, el promedio de la variable de rentabilidad (ROA) fue de $12 \%$ para México, $12 \%$ para Argentina, $8 \%$ en Chile y $10 \%$ en Brasil.

Cuadro 3. Estadísticos descriptivos de las varaibles

\begin{tabular}{|c|c|c|c|c|c|c|c|c|c|c|c|}
\hline & & \multicolumn{10}{|c|}{ País } \\
\hline & & \multicolumn{5}{|c|}{ México } & \multicolumn{5}{|c|}{ Argentina } \\
\hline & & Media & Máximo & Mínimo & $\begin{array}{l}\text { Deswia } \\
\text { ción } \\
\text { std. }\end{array}$ & $\%$ & Media & Máximo & Mínimo & $\begin{array}{l}\text { Desvi } \\
\text { ación } \\
\text { std. }\end{array}$ & $\%$ \\
\hline \multicolumn{2}{|l|}{ WGI } & .48 & .52 & .45 & .02 & & .41 & .44 & .38 & .02 & \\
\hline \multicolumn{2}{|l|}{ Tamaño del consejo } & 13 & 21 & 6 & 4 & & 10 & 17 & 6 & 3 & \\
\hline \multicolumn{2}{|c|}{ Composición del consejo } & .48 & .79 & .20 & .15 & & .27 & .55 & .00 & .18 & \\
\hline \multirow[t]{2}{*}{ Dualidad COB-CEO } & No & & & & & $55.8 \%$ & & & & & $55.4 \%$ \\
\hline & Sí & & & & & $44.2 \%$ & & & & & $44.6 \%$ \\
\hline \multicolumn{2}{|c|}{$\begin{array}{l}\text { Participación femenina en el } \\
\text { consejo }\end{array}$} & .04 & .40 & .00 & .07 & & .00 & .11 & .00 & .01 & \\
\hline \multirow[t]{2}{*}{ Comité de auditoría } & No & & & & & $.0 \%$ & & & & & $.0 \%$ \\
\hline & sí & & & & & $100.0 \%$ & & & & & $100.0 \%$ \\
\hline \multicolumn{2}{|c|}{ Concentración de la propiedad } & .54 & .86 & .13 & .20 & & .53 & .84 & .15 & .14 & \\
\hline \multicolumn{2}{|l|}{ Endeudamiento } & .18 & .60 & .00 & .13 & & .19 & .47 & .02 & .13 & \\
\hline \multicolumn{2}{|l|}{ Edad de la empresa } & 46 & 123 & 4 & 31 & & 47 & 102 & 5 & 31 & \\
\hline \multicolumn{2}{|c|}{ Tamaño de la empresa } & 8.18 & 10.81 & 5.17 & .98 & & 7.57 & 9.63 & 5.97 & 1.10 & \\
\hline $\mathrm{ROA}$ & & .12 & .38 & -.13 & .07 & & .12 & .38 & -.03 & .09 & \\
\hline e-ITGC ponderado & & .72 & .88 & .28 & .10 & & .51 & .84 & .12 & .19 & \\
\hline
\end{tabular}


Cuadro 3. Estadísticos descriptivos de las varaibles (continuación)

\begin{tabular}{|c|c|c|c|c|c|c|c|c|c|c|c|}
\hline & & \multicolumn{9}{|c|}{ País } & \\
\hline & & \multicolumn{5}{|c|}{ Chile } & \multicolumn{5}{|c|}{ Brasil } \\
\hline & & Media & Máximo & Mínimo & $\begin{array}{l}\text { Desvi } \\
\text { ación } \\
\text { std. }\end{array}$ & $\%$ & Mean & Máximo & Mínimo & $\begin{array}{c}\text { Desvia } \\
\text { ción } \\
\text { std. }\end{array}$ & $\%$ \\
\hline \multicolumn{2}{|l|}{ WGI } & .84 & .87 & .82 & .02 & & .52 & .57 & .49 & .03 & \\
\hline \multicolumn{2}{|l|}{ Tamaño del consejo } & 8 & 11 & 5 & 1 & & 9 & 17 & 3 & 3 & \\
\hline \multicolumn{2}{|c|}{ Composición del consejo } & .35 & 1.00 & .00 & .23 & & .30 & .80 & .00 & .19 & \\
\hline \multirow[t]{2}{*}{ Dualidad COB-CEO } & No & & & & & $95.2 \%$ & & & & & $78.1 \%$ \\
\hline & Sí & & & & & $4.8 \%$ & & & & & $21.9 \%$ \\
\hline \multicolumn{2}{|c|}{$\begin{array}{l}\text { Participación femenina en el } \\
\text { consejo }\end{array}$} & .02 & .33 & .00 & .06 & & .06 & .33 & .00 & .09 & \\
\hline \multirow[t]{2}{*}{ Comité de auditoría } & No & & & & & $71.2 \%$ & & & & & $6.8 \%$ \\
\hline & Sí & & & & & $28.8 \%$ & & & & & $93.2 \%$ \\
\hline \multicolumn{2}{|c|}{ Concentración de la propiedad } & .58 & .99 & .31 & .14 & & .55 & 1.00 & .00 & .17 & \\
\hline \multicolumn{2}{|l|}{ Endeudamiento } & .27 & .63 & .06 & .10 & & .31 & .66 & .00 & .15 & \\
\hline \multicolumn{2}{|l|}{ Edad de la empresa } & 62 & 160 & 5 & 36 & & 41 & 125 & 0 & 34 & \\
\hline \multicolumn{2}{|c|}{ Tamaño de la empresa } & 7.76 & 10.68 & 4.97 & 1.16 & & 8.68 & 12.78 & 4.85 & 1.52 & \\
\hline ROA & & .08 & .25 & -.13 & .06 & & .10 & .77 & -.13 & .10 & \\
\hline e-ITGC ponderado & & .59 & .86 & .28 & .10 & & .61 & .88 & .07 & .17 & \\
\hline
\end{tabular}

Fuente: Los autores, información obtenida de los informes anuales.

\subsection{Análisis univariante y multivariable}

El cuadro 4 (panel A), reveló un número de correlaciones significativas entre la variable dependiente $(e-I T G C)$ y las variables independientes. Se observa que el $e-I T G C$ está relacionado de forma significativa con el año de estudio, el país, el índice mundial de gobierno (WGI), el tamaño y composición del consejo, el comité de auditoría y la rentabilidad de la empresa $(R O A)$. Para evaluar la multicolinealidad potencial entre las variables, se analizaron las variables que explican el $e-I T G C$ y se calcularon los factores de inflación de la varianza (VIF), los cuales mostraron estar por debajo de 2 y el nivel de tolerancia por encima de $0.60,+$ lo cual descarto los problemas de correlación y multicolinealidad entre variables (Xiao et al., 2004). El cuadro 4 (panel B) muestra dichos estadísticos. 
118 Nueva Época REMEF (The Mexican Journal of Economics and Finance)

Cuadro 1. Panel A: Resumen de variables y anotaciones en el modelo de regresión

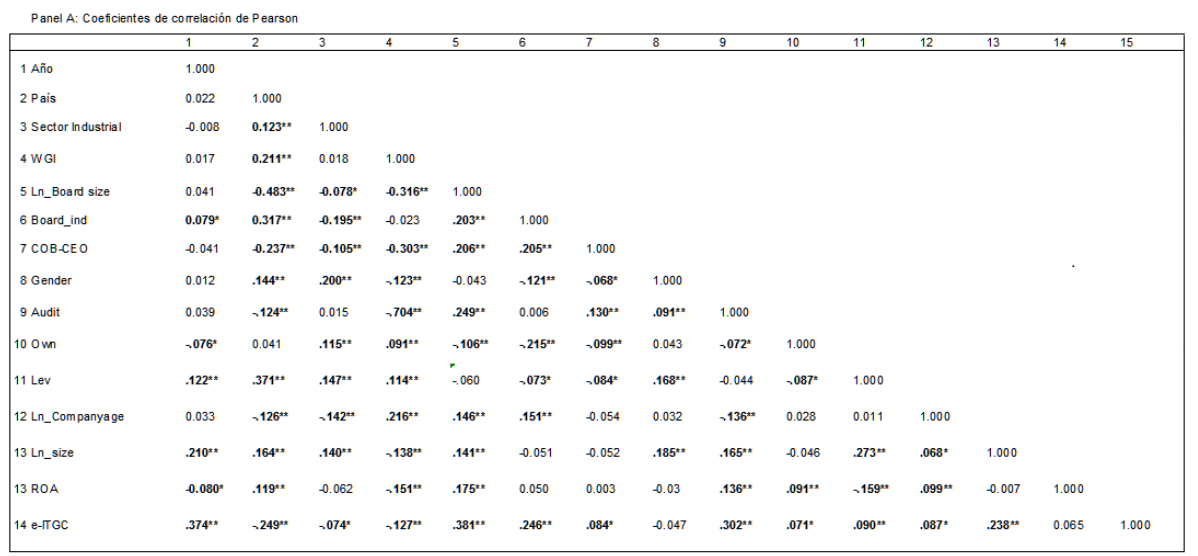

*La correlación es significativa al nivel del 0.05 (bilateral), ${ }^{* *}$ La correlación es significativa an nivel del 0.01 (bilateral)

Cuadro 1. Panel B: Factores de tolerancia e inflación de la varianza

\begin{tabular}{|c|c|c|c|}
\hline Variable & B & VIF & Tolerance \\
\hline (Constant) & -47.498 & & \\
\hline Año & $0.024^{\text {tat }}$ & 1.076 & 0.929 \\
\hline País & -0.019 & 1.734 & 0.577 \\
\hline Sector industrial & $-0.003^{\star}$ & 1.138 & 0.879 \\
\hline WGI & $0.253^{\text {th }}$ & 2.47 & 0.405 \\
\hline Ln_Board & $0.1^{\star x+}$ & 1.555 & 0.643 \\
\hline Board_composition & 0.1 & 1.246 & 0.803 \\
\hline COB-CEO duality & 0.008 & 1.212 & 0.825 \\
\hline Gender of the board & -0.075 & 1.136 & 0.880 \\
\hline Audit & $0.141^{\prime \prime}$ & 2.044 & 0.489 \\
\hline Ownership & 0.036 & 1.104 & 0.906 \\
\hline Leveage & 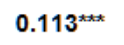 & 1.317 & 0.759 \\
\hline Ln_companyage & -0.003 & 1.196 & 0.836 \\
\hline Size & $0.015^{\ldots+1}$ & 1.266 & 0.790 \\
\hline ROA & 0.043 & 1.105 & 0.905 \\
\hline
\end{tabular}

Fuente Elaboración Propia. 1.- Las variables son definidas en el cuadro 2. 2.Los coeficientes están basados en 832 observaciones, 3.- Los coeficientes son significativos al $\mathrm{p}=.10\left(^{*}\right), \mathrm{p}=.05\left({ }^{* *}\right)$ y $\mathrm{p}=.01\left({ }^{* *}\right)$, se marcan en negrita, 4.- La variable dependiente es el $e-I T G C, 5 .-$ VIF $=$ Factor de inflación de la varianza 
Para comprobar las relaciones entre el $e-I T G C$ y los factores institucionales formales, se realizó el análisis de regresión múltiple. Se adoptó el método de mínimos cuadrados ordinarios con el estimador de los componentes de la varianza (LS-VCE). Éste es considerado un método robusto que estima los componentes de la (co)varianza de forma lineal y permite obtener estimadores no sesgados (Amiri-Simkooei, 2007). El modelo en sí se derivó de la agregación de los cuatro sub-índices del $e-I T G C$, la variables del sistema legal y de gobierno corporativo (tamaño y composición del consejo, dualidad COB-CEO, participación femenina del consejo, presencia del comité de auditoría y concentración de la propiedad), así como las variables de control corporativas (tamaño y edad de la empresa, ROA, endeudamiento, y sector industrial). Las variables que resultaron ser significativas en el modelo fueron: el índice mundial de gobierno (valor de $\mathrm{p}=0.000$ ). Respecto a las variables de gobierno corporativo resultaron significativas el tamaño del consejo (valor de $\mathrm{p}$ $=0.000$ ), la composición del consejo (valor de $\mathrm{p}=0.000$ ), la participación de la mujer en el consejo (valor de $\mathrm{p}=0.077$ ) y la presencia del comité de auditoría (valor de $\mathrm{p}=0.000$ ). El modelo de regresión obtuvo un $\mathrm{r}$ cuadrado de 0.4349. Estos resultados son congruentes con los obtenidos por Vander Bauwhede y Willekens (2008), Barako et al. (2006) y Bonsón y Escobar (2006).

En este contexto, no se rechaza la hipótesis que sostiene una relación positiva entre los factores institucionales formales y el $e-I T G C$ (La Porta et al., 2004; Jaggi y Low, 2000). Se observa que el sistema legal y algunas de las variables de gobierno corporativo incrementan el nivel de transparencia en gobierno corporativo. Particularmente, el tamaño del consejo, su composición y la presencia del comité de auditoría inciden positivamente en dicha relación (Kent y Steward, 2008; Barako et al., 2006; Willekens et al., 2005; Ajinkya et al., 2005). Por su parte, las variables de control significativas en el modelo son el endeudamiento $(\mathrm{p}=0.037)$ y tamaño de la empresa $(\mathrm{p}=0.000)$. Con respecto a la influencia que tienen el sector industrial y año de análisis se observó una menor divulgación de información en los sectores de servicios de consumo, teconologías de información y empresas del sector de energías, mientras que a partir de 2006, el incremento en la transparencia de gobierno corporativo se ha incrementado significativamente en estos países.

Las variables que no son significativas en el modelo son la concentración de la propiedad y la dualidad COB-CEO. Por su parte, no se observó una influencia significativa de la presencia femenina en los consejos de administracion en la decision de divulgar mayor informacion hacia el mercado. Las variables a nivel empresa como la rentabilidad (ROA) y la edad de la empresa no inciden significativamente en el análisis. Estos resultados han sido sustentados por los trabajos realizados por Gandía (2008), Hope et al. (2008) y Oyelere et al. (2003).

\section{Conclusiones}

La transparencia en gobierno corporativo ha cobrado gran relevancia, y ha sido adoptada como una estrategia por parte de los gobiernos y las empresas para mantener la integridad en los mercados financieros. Una extensa literatura ha analizado la posible relación entre el nivel de transparencia en gobierno corporativo y los factores institucionales. Para cuantificar esta información, 
se han propuesto diferentes índices, siendo los más recomendados aquellos que agregan la información de forma dicotómica y sin criterio de ponderación.

En este sentido, nuestro primer objetivo en el trabajo fue realizar un comparativo entre países emergentes Latinoamericanos a través de la construcción de un índice de transparencia en gobierno corporativo que integra la información proporcionada en los sitios web de las empresas y en los informes anuales. El segundo objetivo, fue analizar si los factores institucionales formales referidos al sistema legal y las dimensiones de gobierno corporativo podrían tener una influencia en el e-ITGC, a través del análisis de regresión múltiple.

Los resultados descriptivos muestran diferencias significativas en el nivel de divulgación de información entre el grupo de países analizados. Por un lado, las sociedades cotizadas en México obtienen un $e-I T G C$ del $72 \%$, Argentina apenas obtiene el $51 \%$, mientas que Chile y Brasil un $59 \%$ y $61 \%$ respectivamente. Estos países han centrado en mayor medida sus esfuerzos a la divulgación de información relacionada a ética y conflictos de interés. En cuanto a los factores que inciden en un mayor o menor nivel de transparencia, encontramos al sistema legal, el tamaño y composición del consejo y la presencia del comité de auditoría. El tamaño y endeudamiento de la empresa, el sector industrial al que pertenece y el año de estudio son variables significativas en el estudio. Sin embargo, otras variables como la concentración de la propiedad, la dualidad COB-CEO, la participación femenina en el consejo, la edad de la empresa y la rentabilidad corporativa no son significativas en el análisis. En resumen, estos resultados sugieren que los factores que han sido propuestos en la literatura, y que han recibido sustento empírico como determinantes en el nivel de transparencia en GC, juegan un papel importante en el contexto de estos países.

Nuestro trabajo contribuyó a la literatura en tres aspectos principales: 1) A pesar de que existe un amplio cuerpo de investigación que ha puesto atención en el impacto que tienen las dimensiones de gobierno corporativo sobre el nivel de transparencia, son escasos los estudios que se han enfocado a la divulgación de información sobre gobernanza empresarial en economías emergentes Latinoamericanas; 2) el presente estudio es pionero en comparar cuál ha sido el comportamiento en materia de transparencia en gobierno corporativo e identifica si el sistema legal y las dimensiones de buen gobierno son factores que inciden en el índice de transparencia; 3) los resultados de esta investigación podrían ser un referente para los responsables de emitir las políticas y lineamientos en materia de gobernanza empresarial.

Existen varias limitaciones en este estudio. En primer lugar, el problema subjetivo inherente a la calificación y evaluación de información recogida en los informes anuales de muestra de empresas no puede ser excluido. En segundo lugar, cada elemento que integra el e-ITGC tiene el mismo peso, no existiendo un criterio de ponderación. En tercer lugar, el análisis de regresión lineal no resuelve los problemas de causalidad entre variables.

No obstante, a pesar de las limitaciones antes mencionadas, los resultados son lo suficientemente interesantes como para justificar y ampliar la investigación a otros países. Otra línea futura de investigación interesante es incluir variables institucionales informales como la cultura y estrategia empresarial y su influencia sobre la transparencia en gobierno corporativo. Otra línea interesante, es realizar un estudio longitudinal por sector industrial, lo que 
permitiría analizar la incidencia de los atributos de gobierno corporativo en un sector específico de actividad.

\section{Bibliografía}

Adams, R. y D. Ferreira (2004). Gender Diversity in the Boardroom. European Corporate Governance Institute, Finance Working paper 57, pp. 30.

Ajinkya, B. S. y P. Sengupta (2005). The Association between Outside Directors, Institutional Investors and the Properties of Management Earnings Forecasts. Journal of Accounting Research, 43, pp. 343-376.

Amiri-Simkooei, A. (2007). Least-Squares Variance Component Estimation: Theory and GPS Applications. Tesis doctoralr5 http://www.narcis.nl/publication/RecordID/oai:tudelft.nl:uuid:bc7f8919-1baf-4f02-b115-dc926c5ec090/Language/

Barako, D. G., P. Hancock y H. Izan (2006). Factors Influencing Voluntary Corporate Disclosure by Kenyan Companies. Corporate Governance: An International Review, 14(2), pp. 107-126.

Baysinger, B. y R. Hoskinsson (1990). The Composition of Boards of Directors and Strategic Control: Effects on Corporate Strategy. Academy of Management Review, 15(1), pp. $72-87$.

Bhojraj, S., C. M. Lee y D. K. Oler (2003). What's my Line? A Comparison of Industry Classification Schemes for Capital Market Research. Journal of Accounting Research, 41(5), pp. 745-774.

Boliari, N. y K. Topyan (2007). Conceptualizing Institutions and Organizations: A Critical Approach. Journal of Business \& Economics Research, 5(1), pp. 1-10.

Blackburn, V. L. (1994). The Effectiveness of Corporate Control in the U.S. Corporate Governance: An International Review, 2(4), pp. 196-202.

BM\&FBOVESPA. (06 de Enero de 2011). BMyFBOVESPA. Recuperado el 24 de Enero de 2011, de BMyFBOVESPA: http://www.bmfbovespa.com.br/es-es/noticias/2011/BMFBOVESPA-lanza-el-Indice-de-Gobierno-Corporativo-Trade-IGCT-2011-01-06.aspx? es

Bolsa de Comercio de Santiago. (2011). Metodología de selección y cálculo de índices bursátiles. Santiago de Chile: Bolsa de Comercio de Santiago.

Bonsón, E. y T. Escobar (2006). Digital Reporting in Eastern Europe: An empirical Study. International Journal of Accounting Information Systems, 7, pp. 299-318.

Bushman, R., J. D. Piotroski y A. J. Smith (2004). What Determines Corporate Transparency? Journal of Accounting Research, 42, pp. 207-252.

Bushman, R., J. Piotroski, A. J. Smith (2004). What Determines Corporate Transparency? Journal of Accounting Research, 42, pp. 207-252.

Che Haat, M. H., R. A. Rahman; y S. Mahenthiran (2008). Corporate Governance, Transparency and Performance of Malaysian Companies. Managerial Auditing Journal, 23 (8), pp. 744-778.

Chen, C. J. y B. Jaggi (2000). Association between Independent Non-Executive Directors, Family Control and Financial Disclosures in Hong Kong. Journal of Accounting and Public Policy, 19, pp. 285-310.

Choi, C., Hilton, B. y Millar, C. (2004). Emergent Globalization. London, UK: Palgrave Macmillan.

Chong, A.; J. Guillen; y F. Lopez-de-Silanes (2009). Corporate Governance Reform and Firm Value in Mexico: An Empirical assesment. Journal of Economic Policy Reform, 12(3), pp. 163-188.

Collier, P. (1997). Audit Committees in Smaller UK Companies. En Keasey, K., Wright, M. eds. Corporate Governance: Responsabilities, Rights and Remuneration, pp. 93-120.

Darrough, M. N. y N. M. Stoughton (1990). Financial Disclosure Policy in an Entry Game. Journal of Accounting and Economics, 12 (1-3), pp. 219-243.

Depoers, F. (2000). A Cost-Benefit Study of Voluntary Disclosure: Some Empirical Evidence from French listed Companies. The European Accounting Review, 9(2), pp. 245-263. 
Diamandis, P. F. y A. A. Drakos (2011). Financial Liberalization, Exchange Rates and Stock Prices: Exogenous Shocks in Four Latin America Countries. Journal of Policy Modeling, 33(3), pp. 381-394.

DiMaggio, P. J. y W. W. Powell (1983). The Iron Cage Revisited: Institutional Isomorphism and Collective Rationality in Organizational Fields. American Sociological Review, 48 pp. 147-160.

Djankov, S., R. La Porta, y F. Lopez-de-Silanes (2008). The Law and Economics of Self-Dealing. Journal of Financial Economics, 88 (3), 430-465.

Durnev, A. y E. H. Kim (2005). To Steal or not to Steal: Firm Attributes, Legal environment, and Valuation. The Journal of Finance, 60 (3), pp. 1461-1493.

Ettredge, M., V. Richardson y S. Scholz (2001). The Presentation of Financial Information at Corporate Web Sites. International Journal of Accounting Information System, 2, pp. 149-168.

Fama, E. F. y M. C. Jensen (1983). Separation of Ownership and Control. Journal of Law and Economics, 26(2), pp. 301-325.

Francoeur, C., R. Labelle y B. Sinclair-Desgagné (2008). Gender Diversity in Corporate Governance and top Management. Journal of Business Ethics, 81, pp. 83-95.

Gandía, J. L. (2008). Determinants of Interest-Based Corporate Governance Disclosure by Spanish listed Companies. Online Information Review, 32(6), pp. 791-817.

Gandía, J. L. y T Andrés (2005). E-Gobierno corporativo y transparencia informativa en las sociedades cotizadas españolas: un estudio empírico. Comisión Nacional del Mercado de Valores (Monografías No. 8), pp. 1-47.

Garay, U. y González, M. (2008). Corporate Governance and Firm Value: The case of Venezuela. Corporate Governance: An International Review, 16(3), pp. 194-210.

Globerman, S. y D. Shapiro (2003). Governance Infrastructure and US foreign Direct Investment. Journal of International Business Studies, 34(1), pp. 19-39.

Gul, F. A., B. Srinidhi y A. C. Ng (2011). Does Board Gender Diversity Improve the Informativeness of Stock Prices? Journal of Accounting and Economics, 51(3), pp. 314-338.

Haniffa, R. y T. Cooke (2002). Culture, Corporate Governance and Disclosure in Malaysian Corporations. Abacus, 38, pp. 317-349.

Ho, S. y K. Wong (2001). A Study of the Relationship between Corporate Governance Structures and the extent of Voluntary Disclosure. Journal of International Accounting, Auditing and Taxation, 10, pp. 139-159.

Hodge, F., P. Hopkins y J. Pratt (2006). Management Reporting Incentives and Classification Credibility: The Effects of Reporting Discretion and Reputation. Accounting, Organizations and Society, 31(7), pp. 623-634.

Huse, M. y G. Solberg (2006). Gender-Related boardroom Dynamics: How Scandinavian Women Make and can Make Contributions on Corporate Boards. Women In Management Review, 21(2), pp. 113-130.

Hussainey, K. y B. Al-Najjar (2011). Future-Oriented Narrative reporting: Determinates and Use. Journal of Applied Accounting Research, 12(2), pp. 123-138.

IFAC. (Junio de 2010). International Federation of Accountants. Recuperado el 19 de Enero de 2011, de http://web.ifac.org/download/Business_reporting_article.pdf

IFC. (2010). Navigating through Crisis. A Handbook for Boards. Washington D.C.: International Finance Corporation.

Inchausti, B. G. (1997). The Influence of Company Characteristics and Accounting Regulation. The European Accounting Review, 6, pp. 45-68.

Jaggi, B. y P. Low (2000). Impact of Culture, Market Forces and Legal System on Financial Disclosures. The International Journal of Accounting, 35, PP. 495-519.

Kaufmann, D., Kraay, A. y M. Mastruzzi (2010). The Worldwide Governance Indicators. Methodology and Analytical Issues. Policy Research. Working Paper, PP. 1-31.

Kent, P. y J. Stewart (2008). Corporate Governance and Disclosures on the Transition to International Financial Reporting Standards. Accounting and Finance, 48, pp. 649-671. 
La Porta, R. L. (2006). What Works in Securities Laws? The Journal of Finance, 61(1), pp. 1-32.

La Porta, R., F. López de Silanés, A. Schleifer, y R. Vishny (1998). Law and Finance. Journal of Political Economy, 106, pp. 1113-1155.

Lang, M. y R. Lundholm (1993). Cross-Sectional Determinants of Analyst Ratings of Corporate Disclosures. Journal of Accounting Research, 31, pp. 246-271.

Leal, R. P. y A. L. Carvalhal-da-Silva (2005). Corporate Governance and Value in Brazil (and in Chile). Working Paper. SSRN-id726261, 77.

Lefort, F. y E. Walker (2005). The Effect of Corporate Governance Practices on Company Market Valuation and Payout Policy in Chile. Inter-American Development Bank Working Paper, 515, pp. 1-67.

Leung, S. y B. Horwitz (2004). Director ownership and Voluntary Segment Disclosure: Hong Kong Evidence. Journal of International Financial Management and Accounting, 15, pp. 235-260.

Mangena, M. y V. Tauringana (2007). Disclosure, Corporate Governance and Foreign share Ownership on the Zimbabwe Stock Exchange. Journal of International Financial Management and Accounting, 18(2), pp. 53-85.

Matin de Holan, P. y L. Sanz (2006). Protected by the Family? How Closely held Family Firms Protect Minority Shareholders. Journal of Business Research, 59, pp. 356-359.

McInerney-Lacombe, N., Bilimoria, D. y Salipante, P. (2008). Championing tough issues: How women corporate directors contribute to board deliberations. En S. Vinnicombe, V. Singh, R. J. Burke, D. Bilimoria y M. Huse, (Eds.). Women on corporate boards of directors: Research and practice, 123-139.

Mercer, M. (2004). How Do Investors Assess the Credibility of Managements Disclosures? Accounting Horizons, 18 (3), 185-196.

Mir, M. Z., B. Chatterjee y A. S. Rahaman (2009). Culture and Corporate Voluntary Reporting. Managerial Auditing Journal, 24 (7), pp. 639-667.

McKinnon, J. L. y L. Dalimunthe (1993). Voluntary Disclusure of Segment Information by Australian Diverfied Companies. Accounting y Finance, 33(1), pp. 33-50.

Mundial, E. B. (31 de Diciembre de 2010). Worldwide Governance Indicators. Recuperado el 27 de Enero de 2011, de http://info.worldbank.org/governance/wgi/index.asp

North, D. C. 2005. Understanding the Process of Economic Change, Princeton University Press.

OCDE. (2004). Principios de gobierno corporativo de la OCDE. París: OCDE.

OCDE. (1999). Principios de la OCDE para el gobierno de las sociedades. París: Organización para la Cooperación y el Desarrollo Económico.

Owusu-Ansah, S. (1998). The Impact of Corporate Attributes on the Extent of Mandatory Disclosure and Reporting by Listed Companies in Zimbabwe. The International Journal of Accounting, 33, pp. 605-631.

Oyelere, P., F. Laswad y R. Fisher (2003). Determinants of Internet Financial Reporting by New Zealand Companies. Journal of International Financial Management and Accounting, 14(1), pp. 26-63.

Patelli, L. y A. Prencipe, A. (2007). The relationship between Voluntary Disclosure and Independent Directors in the Presence of a Dominant Shareholder. European Accounting Review, 16(1), pp. 5-33.

Reuters. (3 de Enero de 2011). Reuters. Recuperado el 24 de Enero de 2011, de http://www.reuters.com/article/idARN0312152520110103

Rouf, A. (2011). Corporate Characteristics, Governance Attributes and the Extent of Voluntary Disclosure in Bangladesh. African Journal of Business Management, 5(19), pp. 7836-7845.

Scott, W.R. (2008). Approaching Adulthood: The Maturing of Institutional Theory. Theory and Society. 37(5), pp. 427-442.

Shan, Y. G. y R. P. Mclver (2011). Corporate Governance Mechanisms and Financial Performance in China: Panel data Evidence on Listed non Financial Companies. Asia Pacific Business Review, 17(3), pp. 301-324. 
124 Nueva Época REMEF (The Mexican Journal of Economics and Finance)

Silva, B., D. Azúa; P. Díaz; P. y V. Pizarro (2008). The Influence of Institutional Investors on the Transparency of the Chilean Capital Market. Academia: Revista Latinoamericana de Administración, 40, pp. 54-67.

Simon, S. H. y K. S. Wong (2001). A Study of the Relationship between Corporate Governance Structures and the Extent of Voluntary Disclosure. Journal of International Accounting, Auditing and Taxation, 10(2), pp. 139-156.

Sobhani, F. A.; Y. Zainuddin; A. Amran; M. A. Baten (2011). Corporate Sustainability Disclosure Practices of Selected Banks: A Trend Analysis Approach. African Journal of Business Management, 5(7), pp. 2794-2804.

Tsamenyi, M.; E. Enninful-Adu; y J. Onumah (2007). Disclosure and Corporate Governance in Developing Countries: Evidence from Ghana. Managerial Auditing Journal, 22 (3), pp. 319-334.

Treviño, L.J.; D. E Thomas; y J. Cullen (2008). The Three Pillars of Institutional Theory and FDI in Latin America: An institutionalization Process. International Business Review, 17(1), pp. 118-133.

Vander Bauwhede, H. y M. Willekens (2008). Disclosure on Corporate Governance in the European Union. Corporate Governance: An International Review, 16 (2), pp. 101-115.

Verrecchia, R. E. (1990). Information Quality and Discretionary Disclosure. Journal of Accounting and Economics, 12(4), pp. 365-380.

Watts, R. y J. Zimmerman (1986). Positive Accounting Theory. Englewood Cliffs, NJ: Prentice Hall.

Willekens, M; H. Vander Bauwhede; A. Gaeremynck; y L. Van de Gucht (2005). Internal and External Governance and the Voluntary Disclosure of Financial and Non Financial Performance. 15th National BAA Auditing SIG conference, pp. 1-31.

Xiao, J. Z.; H. Yang; y C. W. Chow (2004). The Determinants and Characteristics of Voluntary Internet-Based Disclosures by Listed Chinese Companies. Journal of Accounting and Public Policy, 23, pp. 191-225. 